\title{
Pion decay constants in dense skyrmion matter
}

\author{
Hee-Jung Lee ${ }^{\mathrm{a}}$
}

Department of Physics Education, Chungbuk National University, Cheongju Chungbuk 361-763, Korea

\begin{abstract}
According to the QCD, the hadronic matter can have various phases with matter density and temperature. In general, when there is phase transition in a matter, it is known that a symmetry in the matter changes. In case of the hadronic matter, the chiral symmetry in the matter is expected to be restored when the matter density (or temperature) increases. The actual order parameter with respect to the chiral symmetry in the hadronic matter is known as the quark condensate from the QCD, but the pion decay constant, corresponding to the radius of the chiral circle, plays the role of the order parameter in an effective field theoretical approach to the QCD. In this paper, by using the skyrmion model which is an effective theory to the QCD, we construct the skyrmion matter as a model of the hadronic matter (nuclear matter) and calculate the pion decay constant in the matter. Because of presence of the matter, the pion decay constant is split into the two components, the temporal component and the spatial component. We discuss the phase transition in the skyrmion matter and behavior of the two components of the decay constant for massless pion with density of the skyrmion matter.
\end{abstract}

\section{References}

1. H.-J. Lee, B.-Y. Park, D.-P. Min, M. Rho, and V. Vento, Nucl. Phys. A723, (2003) 427.

2. H.-J. Lee, B.-Y. Park, M. Rho, and V. Vento, Nucl. Phys. A726, (2003) 69.

3. H.-J. Lee, B.-Y. Park, M. Rho, and V. Vento, Nucl. Phys. A741, (2004) 161.

4. H.-J. Lee and B.-Y. Park, Sae Mulli (The Korean Physical Society) 57, (2008) 61.

\footnotetext{
a e-mail: hjlachungbuk.ac.kr
} 


\title{
Pion decay constants in Dense Skyrmion Matter
}

\author{
Hee-Jung Lee ( Chungbuk Nat'l Univ.)
}

\author{
Helmholtz International Summer
}

School, Dubna, 24 July 2008

\section{Contents}

* Overview

* SU(2) Skyrme Model

* Pion decay constant in Skyrmion Matter

* Results

* Summary and discussion 


\section{Overview}

Properties of Pion in free space :

Mass $\left(m_{\pi}\right)=139 \mathrm{MeV}$

Decay constant $\left(f_{\pi}\right)=93 \mathrm{MeV}$

GMOR Relation : $f_{\pi} m_{\pi}=-m_{q}\langle\bar{q} q\rangle$

$\square \frac{\langle\bar{q} q\rangle_{\rho}}{\langle\bar{q} q\rangle_{0}}=1-\frac{\sum_{\pi N}}{m_{\pi}^{2} f_{\pi}^{2}} \rho$

Properties of Pion in Nuclear

Mattero:? Relation : $f_{\pi}^{* 2} m_{\pi}^{* 2}=-m_{q}\langle\bar{q} q\rangle_{\rho}$

Effective Mass $\left(m_{\pi}^{*}\right)=$ ? MeV

Decay Consatant $\left(f_{\pi}^{*}\right)=$ ? MeV

1. Chiral Perturbation Theory

- H. Yabu et al, PRD 50 (1994) 3549.

- V. Thorsson et al, NPA 589 (1995) 633.

- T. S. Park et al, PLB 409 (1997) 26.

- U. Meissner et al, Ann.Phys297(2002)27.

: Fermi Gas \& Mean Field Approx.

$$
\begin{gathered}
\text { Up to } Q^{2} \\
m_{\pi}^{* 2}=m_{\pi}^{2}-2 \pi \frac{a_{\pi N}^{+}}{m_{R}} \rho, \\
f_{\pi}^{t}=f_{\pi}\left(1-\frac{\rho}{\rho_{0}}(0.26 \pm 0.04)\right), \\
f_{\pi}^{s}=f_{\pi}\left(1-\frac{\rho}{\rho_{0}}(1.23 \pm 0.07)\right) .
\end{gathered}
$$




\section{Optical Potential Theory}

- T. Waas et al, PLB 405 (1997)

- T. Yamazaki et al, PLB 418 (1998)

: S-wave pion- nucleon potential of the deeply bound states in $\mathrm{Pb}$ nucleus observed at GSI

$$
m_{\pi}^{* 2}=m_{\pi}^{2}+2 \omega U_{o p t}(r)
$$

Pion mass increases

- a $7 \%$ at the center $\mathrm{Pb}$ nucleus

- a $3 \%$ in symmetric nuclear matt.

\section{SU(2) Skyrme Model Approach}

- Baryons are appeared as static solitons of the Skyrme Lagrangian.

- Skyrmion matter as a model of nuclear matter

- The pion-matter interactions are generated from the same Skyrme Lagrangian as classical potentials.

- The results contain higher orders in density. 


\section{SU(2) Skyrme model with the trace anomaly from QCD}

- J. Ellis and J. Lanik, PLB150 (1985) 289

- G. E. Brown and M. Rho, PRL 66 (1991) 2720

$$
\begin{aligned}
\mathcal{L}= & \frac{f_{\pi}^{2}}{4}\left(\frac{\chi}{f_{\chi}}\right)^{2} \operatorname{Tr}\left(\partial_{\mu} U^{\dagger} \partial^{\mu} U\right)+\frac{1}{32 e^{2}} \operatorname{Tr}\left(\left[U^{\dagger} \partial_{\mu} U, U^{\dagger} \partial_{\nu} U\right]\right)^{2} \\
& +\frac{f_{\pi}^{2} m_{\pi}^{2}}{4}\left(\frac{\chi}{f_{\chi}}\right)^{3} \operatorname{Tr}\left(U+U^{\dagger}-2\right) \quad f_{\chi}: \text { VEV of } \chi \\
& +\frac{1}{2} \partial_{\mu} \chi \partial^{\mu} \chi-\frac{1}{4} \frac{m_{\chi}^{2}}{f_{\chi}^{2}}\left[\chi^{4}\left(\ln \left(\chi / f_{\chi}\right)-\frac{1}{4}\right)+\frac{1}{4}\right] .
\end{aligned}
$$

The scalar field $(\chi)$ with unity scale dim. and the potential energy generate the trace anomaly in this effective Lagrangian :

- Furnstahl, et al, PRC 52 (1995) 1368

\section{Skyrmion Matter (SM) : Skyrmion Crystal}

- I. Klevanov, NPB 262(1985) 133;

- M. Kugler et al, PRD 40 (1989) 3421.

* At low density : FCC (Face Centered Cubic)

Increasing matter density
Phase transition : chiral symm. restoration

* At high density :

Half Skyrmion CC (Cubic centered) 


\section{- Energy per baryon}

$$
\begin{aligned}
E / B=\frac{1}{4} \int_{B o x} d^{3} x & \left\{\frac{f_{\pi}^{2}}{4}\left(\frac{\chi_{0}}{f_{\chi}}\right)^{2} \operatorname{Tr}\left(\partial_{i} U_{0}^{\dagger} \partial_{i} U_{0}\right)+\frac{1}{32 e^{2}} \operatorname{Tr}\left[U_{0}^{\dagger} \partial_{i} U_{0}, U_{0}^{\dagger} \partial_{j} U_{0}\right]^{2}\right. \\
+ & \left.\frac{f_{\pi}^{2} m_{\pi}^{2}}{4}\left(\frac{\chi_{0}}{f_{\chi}}\right)^{3} \operatorname{Tr}\left(2-U_{0}^{\dagger}-U_{0}\right)+\frac{1}{2} \partial_{i} \chi_{0} \partial_{i} \chi_{0}+V\left(\chi_{0}\right)\right\}
\end{aligned}
$$

Simplified Potential Energy for the scalar

field :

$$
\begin{gathered}
E / B(X, L)=X^{2}\left(E_{2} / B\right)+\left(E_{4} / B\right) \\
+X^{3}\left(E_{m} / B\right)+\left(2 L^{3}\right)\left(X^{4}\left(\ln X-\frac{1}{4}\right)+\frac{1}{4}\right) \\
\chi_{0} / f_{\chi}=X
\end{gathered}
$$

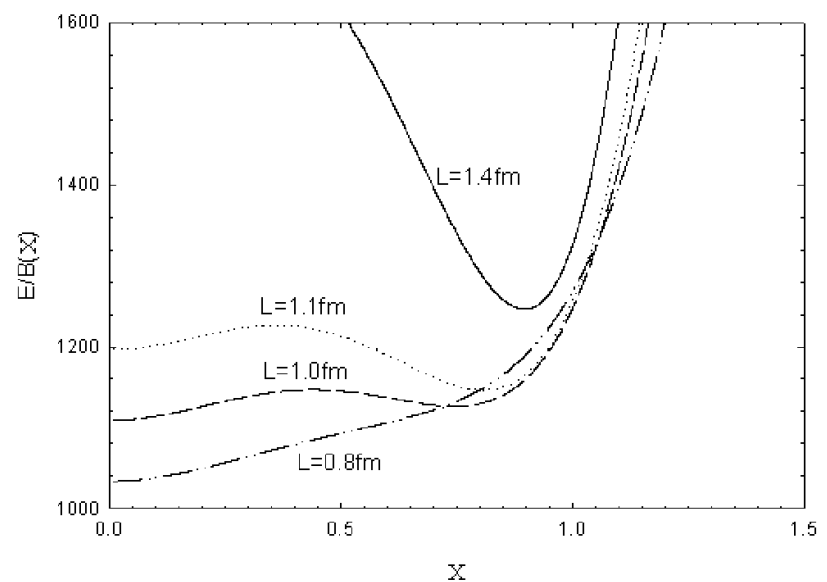

Energy per single skyrmion as a function of the scalar field $\mathrm{X}$ for a given lattice size with the parameter sets B. 


\section{CC}

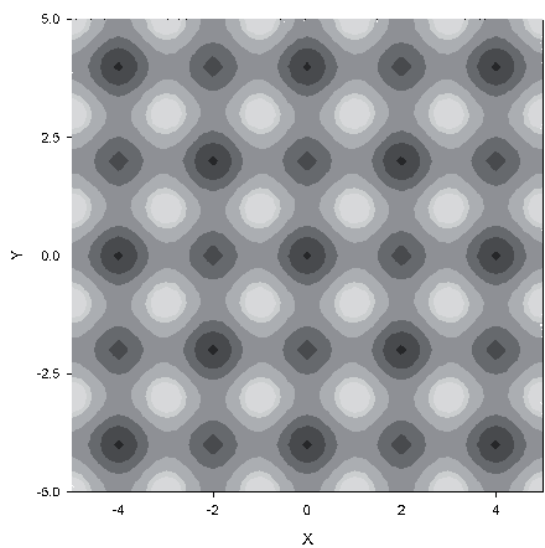

FCC

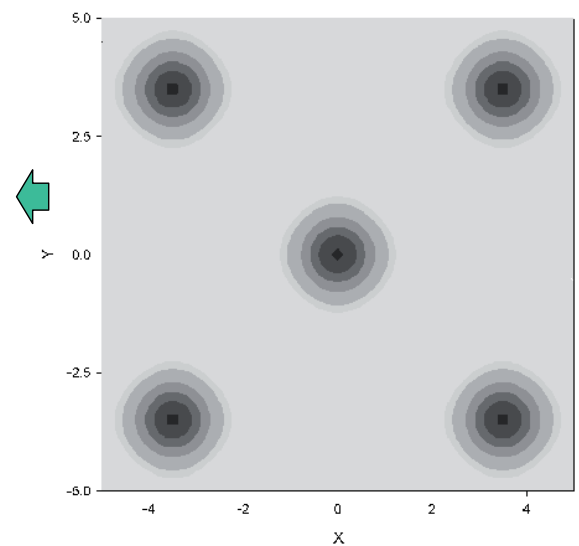

Figure 3: Local baryon number densities at $L=3.5$ and $L=2.0$ with massive pions. For $L=2.0$ the system is (almost) a half-skyrmion in a CC crystal configuration.

Unit of length $(\mathrm{L}): 1 \sim 0.45 \mathrm{fm}$

\section{Phases of skyrmion matter}
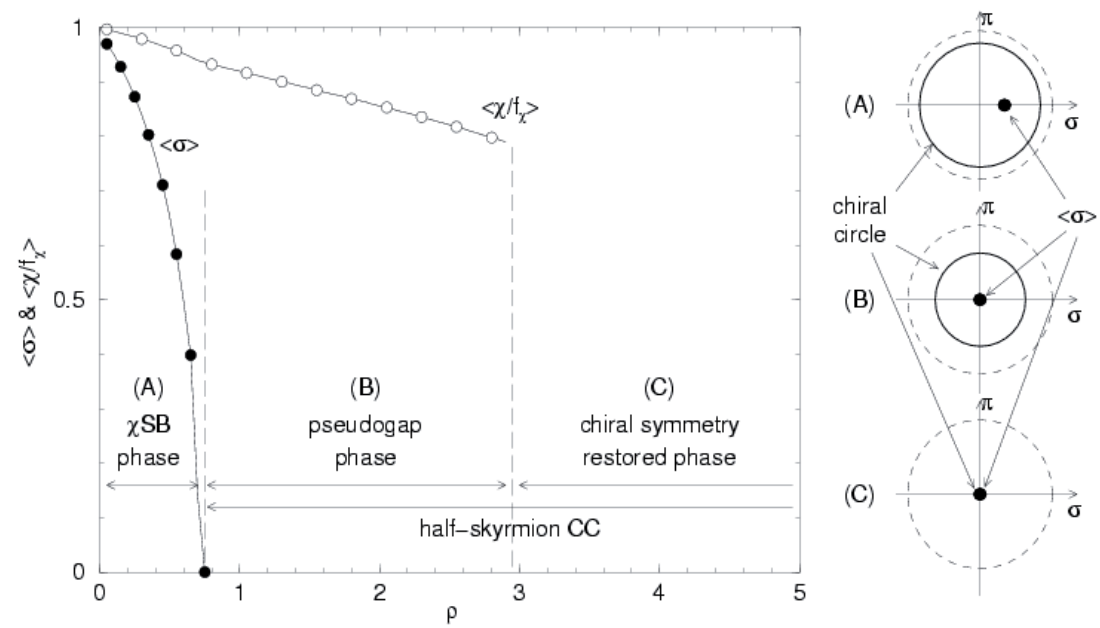

Unit of density : $0.5 \sim \rho_{0}=0.17 \mathrm{fm}^{-3}$ 


\section{Pions and Scalar on Skyrmion Matter}

- Fluctuations on top of the static skyrmion crystal :

$$
U(x)=\sqrt{U_{\pi}(x)} U_{0}(\vec{x}) \sqrt{U_{\pi}(x)}, \quad \chi(x)=\chi_{0}(\vec{x})+\tilde{\chi}(x)
$$

- Lagrangian for pions and the scalar field :

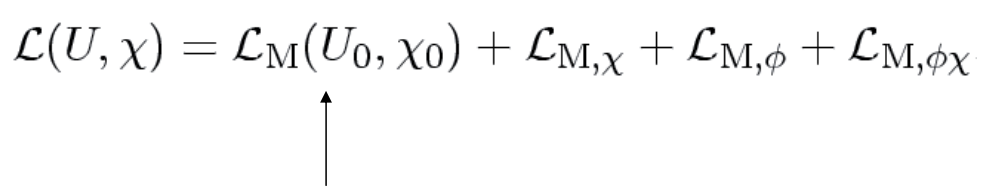

Lagrangian for the static background skyrmion matter

- Lagrangian for pions on the matter :

$$
\begin{gathered}
\mathcal{L}_{\mathrm{M}, \phi}=\frac{1}{2} G_{a b} \dot{\phi}_{a} \dot{\phi}_{b}-\frac{1}{2} H_{a b}^{i j}(\vec{x}) \partial_{i} \phi_{a} \partial_{j} \phi_{b}-\frac{1}{2}\left(\frac{\chi_{0}}{f_{\chi}}\right)^{3} m_{\pi}^{2} \sigma(\vec{x}) \phi_{a}^{2} \\
+\frac{1}{2 f_{\pi}^{2}} \epsilon_{a b c} \partial_{\mu} \phi_{a} \phi_{b} V_{c}^{\mu}(\vec{x})
\end{gathered}
$$

- Lagrangian for the scalar field on the matter :

$$
\begin{gathered}
\mathcal{L}_{\mathrm{M}, \chi}=\frac{1}{2} \partial_{\mu} \chi \partial^{\mu} \chi-\frac{1}{2}\left[m_{\chi}^{2}\left(\frac{\chi_{0}}{f_{\chi}}\right)^{2}\left(1+3 \ln \left(\chi_{0} / f_{\chi}\right)\right)+\frac{2}{f_{\chi}^{2}} \frac{f_{\pi}^{2}}{4} \operatorname{Tr}\left(\partial_{i} U_{0}^{\dagger} \partial_{i} U_{0}\right)\right. \\
\left.+\frac{6 \chi_{0}}{f_{\chi}^{3}} f_{\pi}^{2} m_{\pi}^{2}(1-\sigma(\vec{x}))\right] \chi^{2}
\end{gathered}
$$

- Lagrangian for the interaction between pions and the scalar field on the matter :

$$
\mathcal{L}_{\mathrm{M}, \chi \phi}=\left(\frac{2 \chi_{0}}{f_{\chi}^{2}} \frac{f_{\pi}}{4} \operatorname{Tr}\left(i\left(L_{i}-R_{i}\right) \tau^{a}\right)\right) \chi \partial^{i} \phi_{a}+\left(\frac{3 \chi_{0}^{2}}{f_{\chi}^{3}} \frac{f_{\pi} m_{\pi}^{2}}{2} \operatorname{Tr}\left(i \tau_{a} U_{0}\right)\right) \chi \phi^{a}
$$




\section{Pion decay constants}

two components because of the matter

$$
\begin{array}{lll}
\left\langle\Omega\left|A_{a}^{0}(0)\right| \phi_{b}(q), \Omega\right\rangle & =i \delta_{a b} q^{0} f_{\pi}^{t} & \text { Temporal com. } \\
\left\langle\Omega\left|A_{a}^{i}(0)\right| \phi_{b}(q), \Omega\right\rangle & =i \delta_{a b} q^{i} f_{\pi}^{s} & \text { Spatial com. }
\end{array}
$$

For actual calculation, introducing the external axial vector current $\mathcal{A}_{a}^{\mu}$

$$
L_{A \mathcal{A}}=\left(\frac{\chi_{0}^{2}}{f_{\chi}^{2}}+\frac{2 \chi_{0} \chi}{f_{\chi}^{2}}+\frac{\chi^{2}}{f_{\chi}^{2}}\right)\left[f_{\pi} G^{a b}(\vec{x}) \partial_{\mu} \phi_{b}+A_{M, \mu}^{a}(\vec{x})+\epsilon^{a b c} \frac{\phi_{b}}{f_{\pi}} V_{M, \mu}^{c}(\vec{x})\right] \cdot \mathcal{A}_{a}^{\mu}
$$

with the axial vector current and vector current of SM

$$
A_{M, \mu}^{a}=\frac{i f_{\pi}^{2}}{4} \operatorname{Tr}\left(\left(L_{\mu}^{0}-R_{\mu}^{0}\right) \tau^{a}\right), \quad V_{M, \mu}^{c}(\vec{x})=\frac{i f_{\pi}^{2}}{4} \operatorname{Tr}\left(\left(L_{\mu}^{0}+R_{\mu}^{0}\right) \tau^{c}\right)
$$

\section{Actual calculation}

$$
\begin{aligned}
f_{\pi}^{t} & =-\lim _{\left(q^{0}, \vec{q}\right) \rightarrow\left(m_{\pi}^{* 2}, \overrightarrow{0}\right)} \frac{1}{q_{0}}\left\langle\mathcal{A}_{a}^{0}(q) \mid \phi_{a}(q)\right\rangle_{\text {matter }}, \\
f_{\pi}^{s} & =-\lim _{\left(q^{0}, \vec{q}\right) \rightarrow\left(m_{\pi}^{* 2}, \overrightarrow{0}\right)} \frac{1}{q_{i}}\left\langle\mathcal{A}_{a}^{i}(q) \mid \phi_{a}(q)\right\rangle_{\text {matter }},
\end{aligned}
$$

Relevant vertices for pion decay : from $L_{A \mathcal{A}}$

(a)

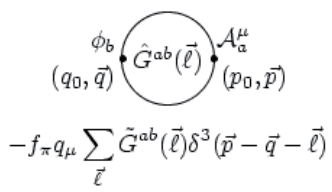

(b)

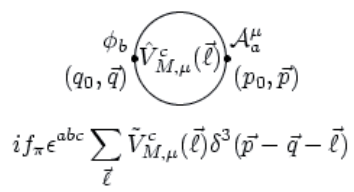

(c)

$$
\begin{gathered}
\left.i q_{0}, \vec{q}\right) \\
\left.i \sum_{\vec{\ell}} P_{\mu}^{a}(\vec{\ell}) \delta^{3}(\vec{\ell})-\vec{q}-\vec{\ell}\right) \\
\mathcal{A}_{a}^{\mu}
\end{gathered}
$$


Vertices from $\mathcal{L}_{\mathrm{M}, \phi}$ and $\mathcal{L}_{\mathrm{M}, \chi \phi}$

(a)

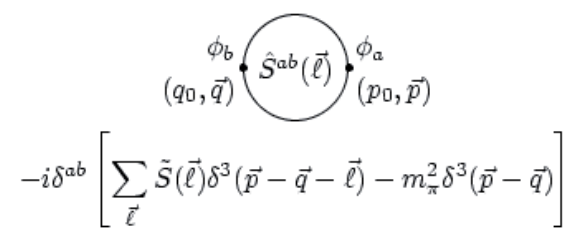

(c)

$$
\begin{gathered}
\phi_{b} \hat{V}_{M, i}^{c}(\vec{\ell}) \cdot g_{\left(p_{0}, \vec{q}\right)}^{\phi_{a}} \\
\epsilon_{a b c} q_{i} \sum_{\vec{\ell}} \tilde{V}_{M, i}^{c}(\vec{\ell}) \delta^{3}(\vec{p}-\vec{q}-\vec{\ell})
\end{gathered}
$$

(b)

$$
\begin{gathered}
i p \cdot q\left[\sum_{\vec{\ell}} \tilde{G}^{a b}(\vec{\ell}) \delta^{3}(\vec{p}-\vec{q}-\vec{\ell})-\delta^{3}(\vec{p}-\vec{q}) \delta^{a b}\right] \\
\hat{G}^{a b}(\vec{\ell}) \cdot \phi_{\left(p_{0}, \vec{p}\right)}^{\phi_{a}}
\end{gathered}
$$

(d)

Pion decay modes : (a) first order approx. (b g) second order approx.

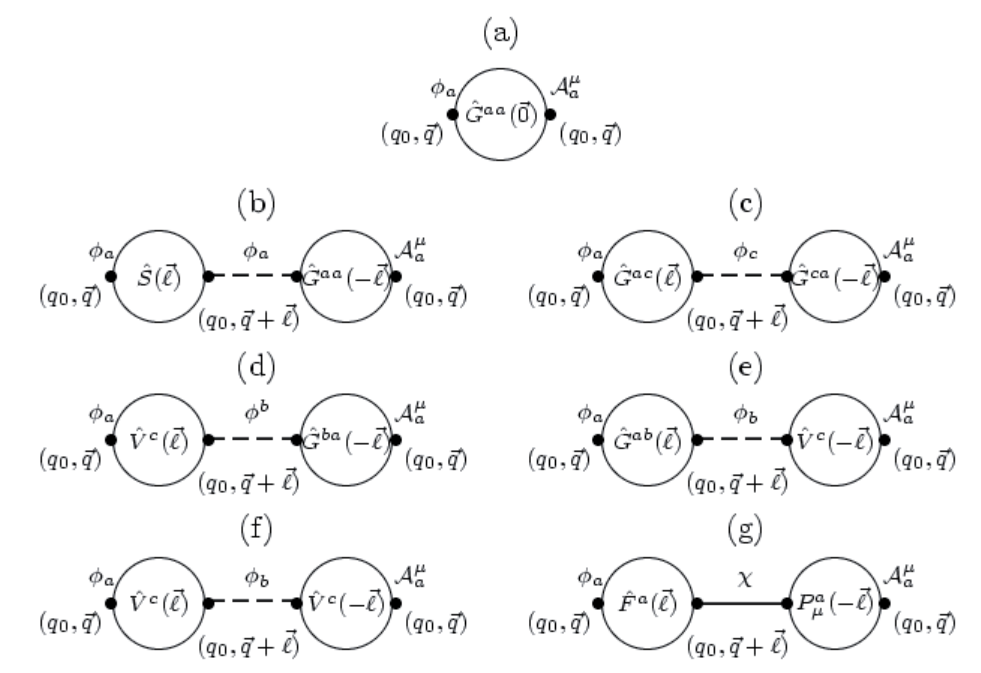




\section{Pion decay constants}

At the first order approx. :

$$
\frac{f_{\pi}^{s}}{f_{\pi}}=\frac{f_{\pi}^{t}}{f_{\pi}}=\sqrt{\hat{G}^{a a}(\overrightarrow{0})}
$$

At the second order approx. :

$$
\begin{aligned}
\frac{f_{\pi}^{t}}{f_{\pi}}= & \sqrt{\hat{G}^{a a}(\overrightarrow{0})}, \\
\frac{f_{\pi}^{s}}{f_{\pi}}= & \sqrt{\hat{G}^{a a}(\overrightarrow{0})} \\
& -\frac{1}{\sqrt{\hat{G}^{a a}(\overrightarrow{0})}} \sum_{\vec{\ell}}\left(\frac{l_{i}^{2} \hat{G}^{a c}(\vec{\ell}) \hat{G}^{c a}(-\vec{\ell})+\hat{V}_{M, i}^{b}(\vec{\ell}) \hat{V}_{M, i}^{b}(-\vec{\ell})}{\vec{\ell}^{2}}+\frac{P_{i}^{a}(\vec{\ell}) P_{i}^{a}(-\vec{\ell})}{\vec{\ell}^{2}+m_{\chi}^{2}}\right)
\end{aligned}
$$

Pion decay constants for the massless pion as a function of density of SM

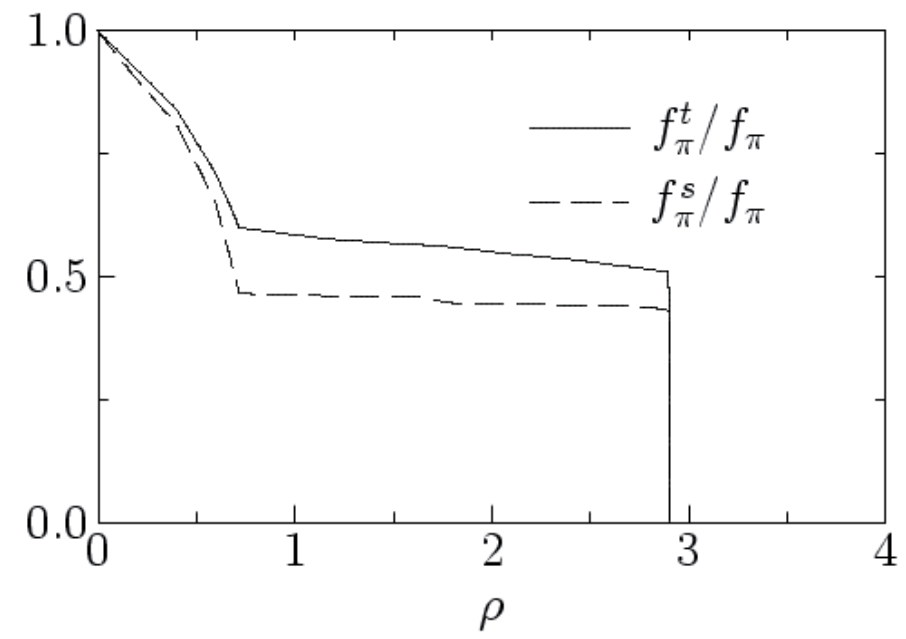

With parameters

$f_{\chi}=240 \mathrm{MeV}$ $m_{\chi}=720 \mathrm{MeV}$

Unit of density : $0.5 \sim \rho_{0}=0.17 \mathrm{fm}^{-3}$ 


\section{Pion velocity}

$$
\begin{aligned}
\mathcal{L}= & \frac{1}{2} G^{a b}(\vec{x}) \partial_{\mu} \varphi_{a} \partial^{\mu} \varphi_{b}+\frac{1}{2} m_{\pi}^{2} \sigma(\vec{x}) \varphi_{a} \varphi_{a} \\
& +\varepsilon_{a b c} \partial_{i} \varphi_{a} \varphi_{b} V_{i}^{c}(\vec{x})
\end{aligned}
$$

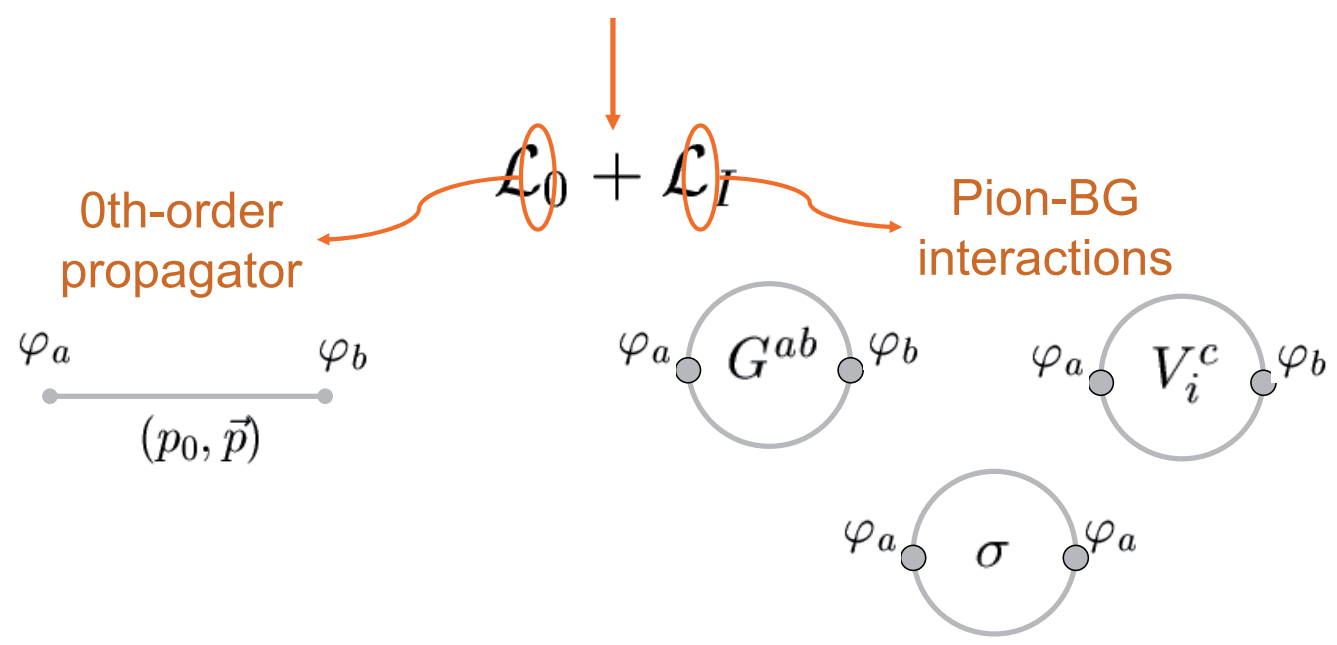

Self energy of the pion and pion propagator in SM

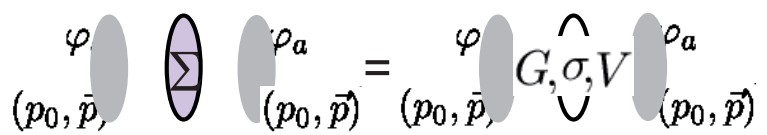

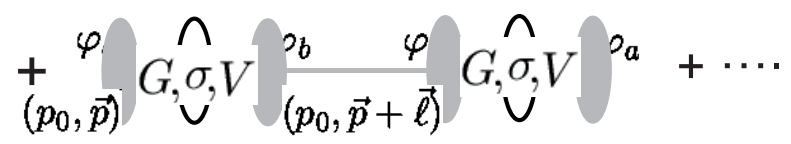




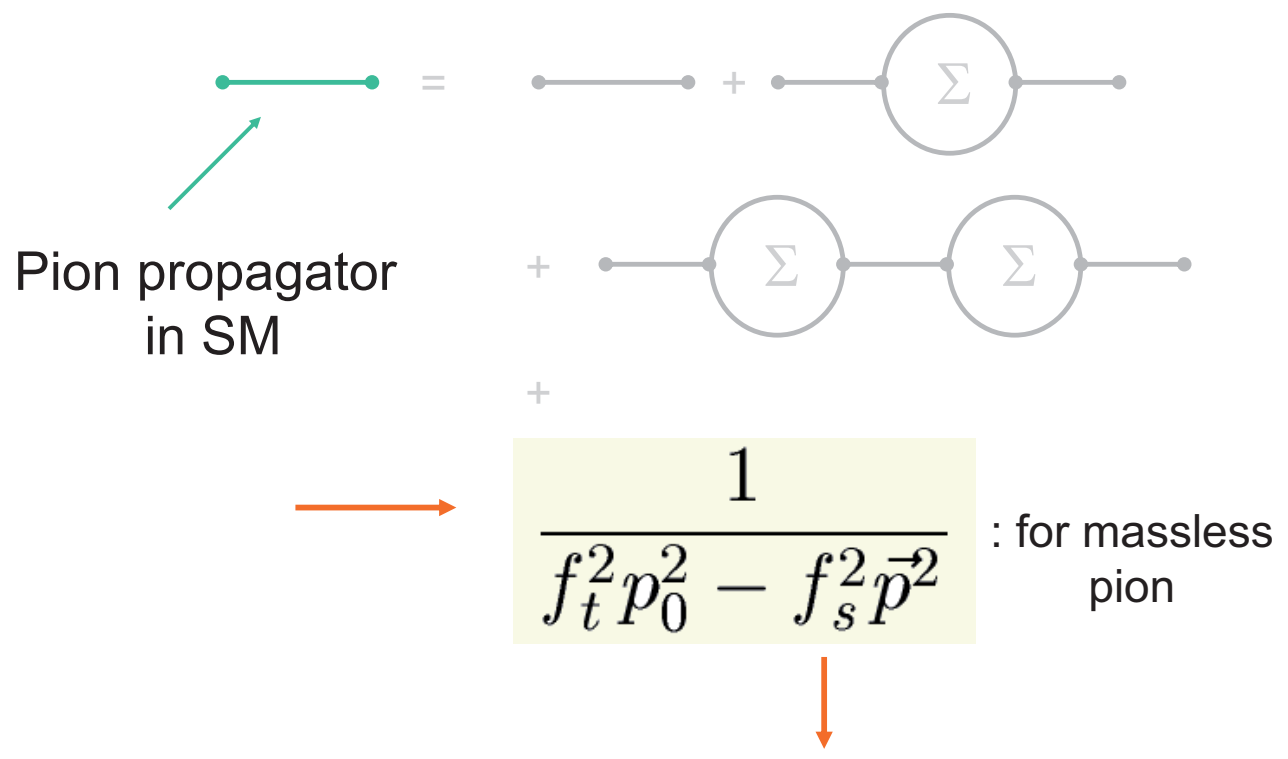

$\underset{\text { In SM }}{\text { Pion velocity }} v_{\pi}=f_{s} / f_{t}$

\section{Pion velocity in medium}

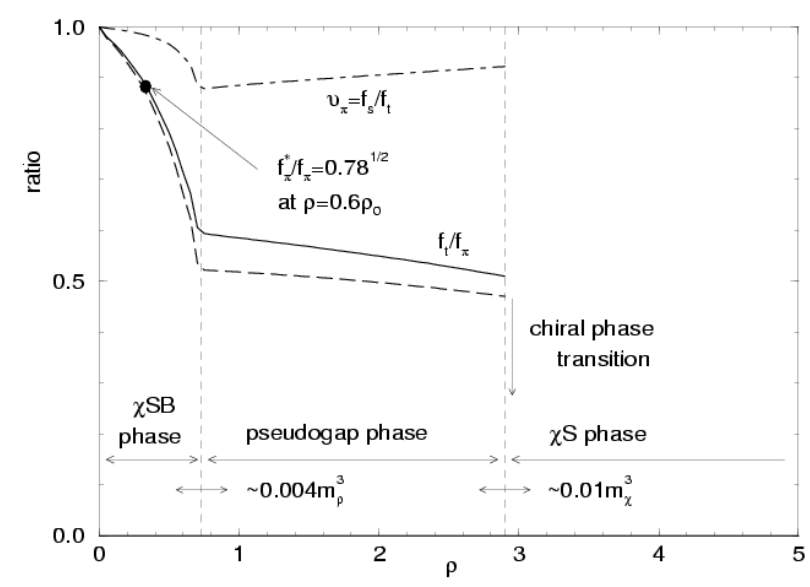

Relation between two kinds

$$
f_{t}=f_{\pi}^{t}, \quad f_{s}=\sqrt{f_{\pi}^{s} f_{\pi}^{t}} .
$$

of constants for the massless pion :

$$
v_{\pi}=f_{s} / f_{t}=\sqrt{f_{\pi}^{s} / f_{\pi}^{t}}
$$




\section{Summary and .....}

1. Phase transition with respect to chiral symmetry happens with density in skyrmion matter.

2. Decay constants of pion decrease with density of skyrmion matter.

3. Velocity of pion decreases to minimum and returns the value in free space when chiral sym. is restored.

4. Relation in two kinds of constants for massive pion?

5. This approach would be too simple to be a real physical situation.

: need to quantize skyrmions in skyrmion matter.

6. Heating skyrmion matter, what happens? 